\title{
Human telomerase prolongs the life span of bovine mammary epithelial cells
}

\author{
W.-J. Sai ${ }^{1,2}$, L.-N. Sui ${ }^{1}$, X.-R. Peng ${ }^{1}$, Y.-G. Wang ${ }^{1}$, S.-Y. Peng ${ }^{1}$ \\ and Y. Zhang ${ }^{1,3}$ \\ ${ }^{I}$ Institute of Biotechnology, Northwest A \& F University \\ Yangling Shaanxi 712100, P.R. China \\ ${ }^{2}$ College of Animal Science and Technology, Xinjiang Shihezhi University \\ Xinjiang Shihezhi 832003, P.R. China
}

\begin{abstract}
The aim of this study was to determine whether the human telomerase catalytic reverse transcriptase (hTERT) could express in bovine mammary epithelial cells (BMEC) and to examine the effects of hTERT on the life span and growth characteristics of the BMEC. We tested one of the hTERT-transferred cell lines (HBM3), the Western Blotting Assay showed that HBM3 had telomerase activity, moreover, HBM3 showed a prolonged life span and demonstrated extensive proliferative capacity as evidenced by their ability to continuously grow even when seeded at low dilutions. In addition, the morphologic features seen in normal BMEC were likewise retained by HBM3, changes associated with neoplastic transformation like anchorage-independent growth and karyotypic instability were not observed.
\end{abstract}

KEY WORDS: bovine, mammary, epithelial cell, telomerase, life span

\section{INTRODUCTION}

To research the developmental and nutritional regulation of the bovine mammary gland, in vitro models of mammary cell lines are essential supplemental tools. However, normal BMEC has a limited proliferate life span in culture and undergo only a finite number of divisions. The cellular senescence may be a limiting factor for the application of BMEC in animal biotechnology.

Telomeres are essential elements that protect chromosomal ends from nuclease degradation and improper recombination, thereby contributing to genome stability

\footnotetext{
${ }^{3}$ Corresponding author: e-mail: zhangyong0085@yahoo.com.cn
} 
(Hackett et al., 2001). However, telomeric repeats are progressively lost each time somatic cells division. Telomeres can be elongated by a telomerase catalytic reverse transcriptase (TERT) and then prolong cell life span (Bodnar et al., 1998). Telomerase-immortalized sheep foetus fibroblast cell line (Cui et al., 2003) and bovine germ stem cell line (Bi et al., 2007) had been obtained.

This study was the first research to determine whether the human telomerase catalytic reverse transcriptase (hTERT) could prolong the life span of bovine mammary epithelial cells (BMEC).

\section{MATERIAL AND METHODS}

\section{Preparation and transfection of cells}

All chemicals were obtained from Sigma (St. Louis, MO, USA) unless otherwise stated. BMEC cells were prepared as previously described (Huynh et al., 1991). Plasmid pETE (pEGFP-hTERT) contains hTERT gene, G418-resistant gene and pEGFP gene driven by CMV promoter. The BMEC cells were cultured in 24-well plate and were lipid transferred by linearized plasmid PETE. The cells with the expression of hTERT were detected by green fluorescence and selected by G418, then were plated into 96-well plates at 50 cells per well in $0.1 \mathrm{ml}$ culture medium. Drug selection continued until the G418-resistant colonies were obtained.

\section{Proliferative capacity and serum-dependency}

For the analysis of proliferative capacity, cells from one of hTERT- transferred bovine mammary cell lines (HBM3) were plated into 96-well plates at 5 cells per well and cultured until 100\% confluence. For the analysis of serum-dependency, cells form HBM3 were kept in serum-deprivation medium and the culture medium was changed for every $48 \mathrm{~h}$.

\section{Telomerase western bloting analysis}

Proteins from HBM3 were extracted in $1 \times$ SDS-PAGE sample loading buffer. Total cell proteins were resolved by SDS-PAGE, then were transferred to PVDF membrane and probed with a anti-hTERT (sc-7212; Santa Cruz, USA) for HBM3 (17th passage). Secondary antibody detection was performed followed by visualization using the BM-chemiluminescence bloting substrate. The breast carcinoma cell line SUM149 and BMEC were used as positive control and negative control, respectively. 


\section{Genetic stability}

Metaphasic chromosomes of HBM3 (55th passage) were prepared as previously described (Verma, 1999). Imaging and karyotyping were performed using the Applied Imaging System (Applied Imaging Corp., Santa Clara, CA). For the anchorage-independent assay, the cells from HBM3 were plated into $0.3 \%$ Sealaque agarose in cell culture medium supplemented with $20 \%$ FBS. An under layer of $1 \%$ agarose was used to prevent spread of cells. Colonies were visualized after 21 days using the MTT assay. The breast carcinoma cell line SUM149 and BMEC were used as positive control and negative control, respectively. Each softagar assay was performed at least three times.

\section{RESULTS}

Cellular senescence was observed when the normal BMEC was maintained to the 15th passage (Figure 1A). We tested one of the hTERT-transferred cell lines HBM3, the HBM3 demonstrated extensive proliferative capacity as evidenced by their ability to continuously grow even when seeded at low dilutions, moreover, we maintained the HBM3 culture beyond the passage 65 and cellular senescence was not observed (Figure 1B). Other growth characteristics of normal BMEC, such as contact inhibition and serum-dependency growth were still remained by HBM3.

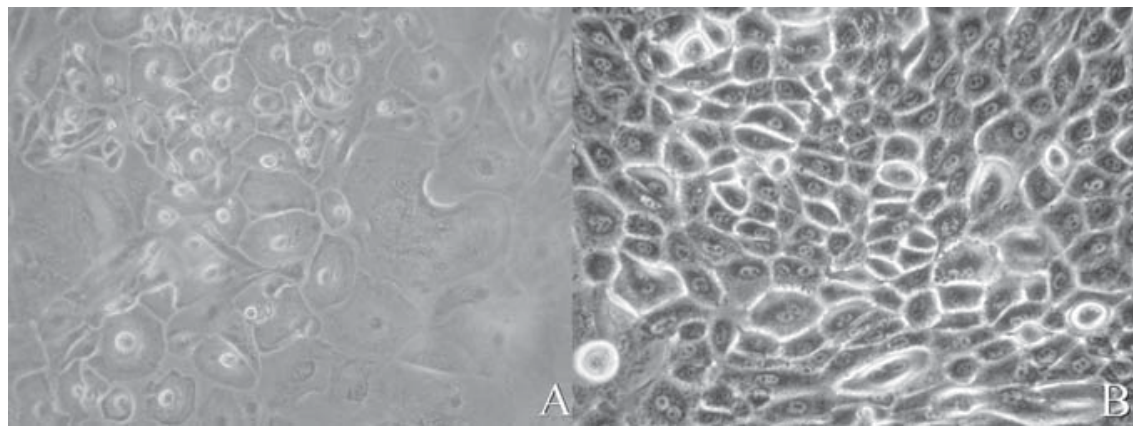

Figure 1. A. Cellular sentence of BMEC with condensed cell nuclei at the 15 th passage. B. Cell morphology of hTERT-transferred HBM3 at the 60th passage

Western Bloting Assay identified hTERT expression in HBM3 cell line and the SUM149 cell line, hTERT expression was not testable in the freshly isolated BMEC by Western Bloting. 
Genetic stability of the high passage of HBM3 was examined by karyotype and the results showed that the high passages of HBM3 had normal karyotype with no structural abnormalities. Moreover, the ability of HBM3 to form colonies in soft-agar was also tested. As shown in Table 1, the HBM3 was not able to form any visible colonies while the breast carcinoma cells SUM149 readily grew and formed $141 \pm 13$ colonies (Table 1).

Table1. Quantification of anchorage-independent growth of different cell lines

\begin{tabular}{cc}
\hline Cell lines & No. of soft-agar colonies \\
\hline BMEC & 0 \\
HBM3 & 0 \\
SUM149 & $141 \pm 13$ \\
\hline
\end{tabular}

values are mean $\pm \mathrm{SE}$ from three independent experiments

\section{DISCUSSION}

There are several ways to obtain immortalized mammary cell lines, one is the use of SV40T (Huynh et al., 1991), or to obtain spontaneously transformed malignant mammary epithelial cell line in vitro culture (Bandyopadhyay et al., 1998). It has been reported previously that expression of hTERT in bovine adrenocortical cells resulted in cell immortalization without affecting their efficiency of transplantation (Thomas et al., 2000). For the research of nutritional regulation of the bovine mammary gland, in this study, we first endeavored to immortalize BMEC by transferring hTERT.

The Western Bloting Assay identified hTERT expression in HBM3. The results indicated that hTERT was compatible with BMEC and any other telomeraserelated factors required for functional telomerase activity in these cells.

Cellular senescence was observed when BMEC was subcultured to the 15th passage (Figure 1A), the expression of hTERT in HBM3 prolonged the cell life span, we maintained HBM3 to the 65 th passage and the cellular senescence was not observed (Figure 1B), moreover, HBM3 showed extensive proliferative capacity. HBM3 still exhibited serum-dependency growth and remained karyotypically stable with no cancer-associated change even after prolonged culturing. The results suggested that expression of hTERT might be a practical way for immortalizing BMEC.

\section{CONCLUSIONS}

It's concluded that the human telomerase catalytic reverase transcriptase (hTERT) could express in bovine mammary epithelial cells and the expression of hTERT resulted in an extended life span in HBM3. 


\section{REFERENCES}

Bandyopadhyay A., Cibull M.L., Sun L.Z., 1998. Isolation and characterization of a spontaneously transformed malignant mouse mammary epithelial cell line in culture. Carcinogenesis 19, 19071911

Bi C.M., Zhang S.Q., Zhang Y., Peng S.Y., Wang L., An Z.X., Qi A., Lv N., 2007. Immortalization of bovine germ line stem cells by c-myc and hTERT. Anim. Reprod. Sci. 100, 371-378

Bodnar A.G., Ouellette M., Frolkis M., Holt S.E., Chiu C.P., Morin G.B., Harly G.B., Shay J.W., Lichtsteiner S., Wright W.E., 1998. Extension of life-span by introduction of telomerase into normal human cells. Science 279, 349-352

Cui W., Wylie D., Aslam S., Dinnyes A., King T., Wilmut I., Clark A.J., 2003. Telomeraseimmortalized sheep fibroblasts can be reprogrammed by nuclear transfer to undergo early development. Biol. Reprod. 69, 15-21

Hackett J.A., Feldser D.M., Greider C.W., 2001. Telomere dysfunction increases mutation rate and genomic instability. Cell 106, 275-286

Huynh H.T., Robitaille G., Jeffrey D., 1991. Establishment of bovine mammary epithelial cells (MAC-T): An in vitro model for bovine lactation. Exp. Cell Res. 197, 191-199

Thomas M., Yang L., Hornsby P.J., 2000. Formation of functional tissue from transplanted adrenocortical cells expressing telomerase reverse transcriptase. Nat. Biotechnol. 18, 39-42

Verma R.S., 1999. Human Cytogenetics: Manual of Basic Techniques. Pergamon, New York 